\title{
GROUPS OF UNITARY COMPOSITION OPERATORS ON HARDY-SMIRNOV SPACES
}

\author{
GAJATH GUNATILLAKE, MIRJANA JOVOVIC, AND WAYNE SMITH \\ (Communicated by Pamela B. Gorkin)
}

\begin{abstract}
Let $\Omega$ be an open simply connected proper subset of the complex plane. We identify, up to isomorphism, which groups are possible for the group of unitary composition operators of a Hardy-Smirnov space defined on $\Omega$. We also study the relationship between the geometry of $\Omega$ and the corresponding group.
\end{abstract}

\section{INTRODUCTION}

Let $\Omega$ be an open simply connected proper subset of the complex plane. The Hardy-Smirnov space $H^{2}(\Omega)$ is the Hilbert space of functions $F$ analytic on $\Omega$ such that the integrals of $|F|^{2}$ over the images of the circles $|z|=r, 0<r<1$, under a Riemann map from the unit disc $\mathbb{D}$ onto $\Omega$ are uniformly bounded; see 3, Chapter 10] or [7, p. 63]. A different choice of Riemann map onto $\Omega$ results in the same space of functions. The classical Hardy space $H^{2}$ of analytic functions on the unit disc corresponds to the choice $\Omega=\mathbb{D}$.

If $f \in H^{2}(\Omega)$ and $\phi$ is an analytic self map of $\Omega$, then the composition operator induced by $\phi$ on $H^{2}(\Omega)$, denoted by $C_{\phi}$, is the linear operator defined by

$$
C_{\phi}(f)=f \circ \phi .
$$

Such operators on $H^{2}(\Omega)$ are studied in [7. Also, composition operators on a Hardy space of a half-plane are studied in [5, 6]. It is elementary that the collection of all unitary operators on a Hilbert space forms a group with group operation the composition of operators. It is not difficult to show that the set of unitary composition operators on $H^{2}(\Omega)$ also forms a group, which we denote by $\mathcal{U}_{\Omega}$ (see Lemma 2.3).

In the case that $\Omega=\mathbb{D}$, it is known that unitary composition operators on $H^{2}$ are precisely those induced by rotations of $\mathbb{D}$, i.e. by the maps $\phi_{\lambda}(z)=\lambda z$, where $|\lambda|=1$; see, for example, [1, Theorem 6]. Moreover since $C_{\phi_{\lambda}} C_{\phi_{\eta}}=C_{\phi_{\lambda \eta}}, \mathcal{U}_{\mathbb{D}}$ is isomorphic to the unit circle with ordinary multiplication of complex numbers.

Our goal in this paper is to show how the geometry of $\Omega$ determines $\mathcal{U}_{\Omega}$ and to identify, up to isomorphism, what groups are possible. In Section 4 it is shown that if $\mathcal{U}_{\Omega}$ is of finite order $n$, then $\Omega$ has $n$-fold symmetry and $\mathcal{U}_{\Omega}$ is isomorphic to $\mathbb{Z}_{n}$. The case that $\mathcal{U}_{\Omega}$ is an infinite group is studied in Section 5 . In Theorem 5.10

Received by the editors July 19, 2013 and, in revised form, December 16, 2013.

2010 Mathematics Subject Classification. Primary 47B33; Secondary 30H10.

Key words and phrases. Composition operator, unitary operator, Hardy-Smirnov space.

The first author would like to thank the University of Hawaii at Manoa for its generosity in hosting him during the collaboration. 
we show that when $\mathcal{U}_{\Omega}$ is infinite, it is isomorphic to one of just five groups. Our results about the geometry of $\Omega$ leading to these groups are summarized in Theorem 5.11. In particular, we show that $\mathcal{U}_{\Omega}$ is isomorphic to the unit circle with usual multiplication if and only if $\Omega$ is a disc.

\section{BACKGROUND MATERIAL}

Throughout this paper $\Omega$ will represent an open simply connected proper subset of the complex plane. Let $\gamma$ be a Riemann map that takes $\mathbb{D}$ onto $\Omega$. For $0<r<1$ let $\Gamma_{r}$ be the curve in $\Omega$ defined by $\Gamma_{r}=\gamma(\{|z|=r\})$. The set of functions analytic on $\Omega$ for which

$$
\sup _{0<r<1} \int_{\Gamma_{r}}|f(w)|^{2}|d w|<\infty
$$

is a Hardy-Smirnov space on $\Omega$. We denote this space by $H^{2}(\Omega)$ and refer to it simply as a Hardy space on $\Omega$. The functional $\|\cdot\|_{\Omega}$ defined on $H^{2}(\Omega)$ by $\|f\|_{\Omega}=\left(\sup _{0<r<1}(1 / 2 \pi) \int_{\Gamma_{r}}|f(w)|^{2}|d w|\right)^{1 / 2}$ is a norm on $H^{2}(\Omega)$ (see [7, p. 63]).

As mentioned above, $H^{2}(\mathbb{D})$ is the classical Hardy space $H^{2}$. For an arbitrary $\Omega$ it turns out that $H^{2}(\Omega)$ is isometrically isomorphic to $H^{2}$.

Theorem A. Suppose $f$ is holomorphic on $\Omega$. Then $f \in H^{2}(\Omega)$ if and only if $(f \circ \gamma)\left(\gamma^{\prime}\right)^{1 / 2} \in H^{2}$. The map $V$ given by $V(f)=(f \circ \gamma)\left(\gamma^{\prime}\right)^{1 / 2}$ is a linear isometry from $H^{2}(\Omega)$ onto $H^{2}$.

For a proof see [7, p. 63] or [3, p. 169]. Using $V$, we can define a function $\langle\cdot, \cdot\rangle_{\Omega}: H^{2}(\Omega) \times H^{2}(\Omega) \rightarrow \mathbb{C}$ by

$$
\langle f, g\rangle_{\Omega}=\langle V(f), V(g)\rangle,
$$

where $\langle\cdot, \cdot\rangle$ is the inner product in $H^{2}$. Since $V$ is an isometry it follows that $\langle f, f\rangle_{\Omega}=\|V(f)\|_{H^{2}}^{2}=\|f\|_{\Omega}^{2}$. Thus $H^{2}(\Omega)$ is a Hilbert space with respect to the inner product $\langle\cdot, \cdot\rangle_{\Omega}$.

Suppose $C_{\phi}$ is a composition operator on $H^{2}(\Omega)$. If $g \in H^{2}$ and $z \in \mathbb{D}$, then

$$
V C_{\phi} V^{-1}(g)(z)=\left(\frac{\gamma^{\prime}(z)}{\gamma^{\prime}(\varphi(z))}\right)^{1 / 2} g(\varphi(z)),
$$

where $\varphi=\gamma^{-1} \circ \phi \circ \gamma$. Let $\psi(z)=\left(\gamma^{\prime}(z) / \gamma^{\prime}(\varphi(z))\right)^{1 / 2}$. Then $V C_{\phi} V^{-1}(g)=C_{\psi, \varphi}(g)$, where $C_{\psi, \varphi}(g)=\psi \cdot g \circ \varphi$. Such an operator is called a weighted composition operator. Moreover, $C_{\phi}$ on $H^{2}(\Omega)$ is isometrically similar to the weighted composition operator $C_{\psi, \varphi}$ on $H^{2}$ (see [7, p. 66]).

Lemma 2.1. If $C_{\phi}$ is invertible, then $\phi$ is an automorphism of $\Omega$ and $C_{\phi}^{-1}=C_{\phi^{-1}}$.

Proof. Clearly, $C_{\phi}$ is invertible if and only if $C_{\psi, \varphi}=V C_{\phi} V^{-1}$ is invertible on $H^{2}$, where $\varphi=\gamma^{-1} \circ \phi \circ \gamma$ and $\psi(z)=\left(\gamma^{\prime}(z) / \gamma^{\prime}(\varphi(z))\right)^{1 / 2}$. From Theorem 2.0.1 of [4] it follows that $C_{\psi, \varphi}$ is invertible if and only if $\varphi$ is an automorphism of $\mathbb{D}$ and $\psi$ and $1 / \psi$ are in $H^{\infty}$. Moreover $C_{\psi, \varphi}^{-1}=C_{1 / \psi \circ \varphi^{-1}, \varphi^{-1}}$. It follows from $\varphi=\gamma^{-1} \circ \phi \circ \gamma$ that $\phi$ is an automorphism of $\Omega$. If $g \in H^{2}(\Omega)$, then a direct computation yields

$$
V^{-1} C_{\psi, \varphi}^{-1} V(g)=g \circ \phi^{-1} \text {. }
$$

Thus $C_{\phi^{-1}}$ is a bounded operator on $H^{2}(\Omega)$ and $C_{\phi}^{-1}=C_{\phi^{-1}}$. 
Interestingly, for certain $\Omega$ it is possible for an automorphism of $\Omega$ to induce a bounded composition operator that is not invertible 8 .

Lemma 2.2. Suppose that $U$ is a bounded operator on $H^{2}(\Omega)$. Then $U$ is unitary if and only if $V U V^{-1}$ is unitary on $H^{2}$.

Proof. Let $T=V U V^{-1}$. Since $V$ is an isometry it is clear that $T$ is an isometry if and only if $U$ is an isometry. Also, $T$ is invertible if and only if $U$ is invertible.

Lemma 2.3. The set of all unitary composition operators of $H^{2}(\Omega)$ is a group under the composition of operators.

Proof. Since $C_{\phi}^{-1}=C_{\phi^{-1}}$ and $C_{f} \circ C_{g}=C_{g \circ f}$ the result follows.

\section{Notation}

- The group of unitary composition operators of $H^{2}(\Omega)$ is denoted by $\mathcal{U}_{\Omega}$.

- The group of the unit circle with usual multiplication is denoted by $(\mathbb{T}, \cdot)$.

- The group of real numbers with usual addition is denoted by $(\mathbb{R},+)$.

- The group of integers with usual addition is denoted by $(\mathbb{Z},+)$.

- $\{1,-1\} \rtimes \mathbb{R}$ is used to denote the semidirect product of the multiplicative group $\{1,-1\}$ and $(\mathbb{R},+)$ with group operation

$$
\left(a_{1}, t_{1}\right) *\left(a_{2}, t_{2}\right)=\left(a_{1} a_{2}, a_{2} t_{1}+t_{2}\right)
$$

where $a_{1}, a_{2} \in\{1,-1\}$ and $t_{1}, t_{2} \in \mathbb{R}$.

- $\{1,-1\} \rtimes \mathbb{Z}$ denotes the subgroup of $\{1,-1\} \rtimes \mathbb{R}$ where the second coordinate is in $\mathbb{Z}$.

- Suppose that $f$ is a self map of the set $\Omega$. If $n \in \mathbb{N}$, then $f_{n}$ is the composition of $f$ with itself $n$ times. If $f$ is invertible and $m$ is a negative integer, then $f_{m}=f_{|m|}^{-1}$. Also $f_{0}$ denotes the identity map.

We leave it to the reader as an easy exercise to show that each of the five groups $(\mathbb{T}, \cdot),(\mathbb{R},+),(\mathbb{Z},+),\{1,-1\} \rtimes \mathbb{R}$ and $\{1,-1\} \rtimes \mathbb{Z}$ belongs to a different isomorphism class.

\section{UNITARY OPERATORS}

We begin our work by classifying those symbols $\phi$ which induce unitary composition operators on $H^{2}(\Omega)$.

Definition 3.1. Let $\mathcal{U} \mathcal{A}_{\Omega}=\left\{\phi: C_{\phi}\right.$ is unitary on $\left.H^{2}(\Omega)\right\}$.

Theorem 3.2. Suppose that $\phi$ is an analytic self map of $\Omega$. Then $\phi \in \mathcal{U} \mathcal{A}_{\Omega}$ if and only if there exist $\mu, c \in \mathbb{C},|\mu|=1$, such that $\phi(w)=\mu w+c$ is an automorphism of $\Omega$.

Proof. First suppose that $\phi(w)=\mu w+c,|\mu|=1, c \in \mathbb{C}$, is an automorphism of $\Omega$. Let $\varphi=\gamma^{-1} \circ \phi \circ \gamma$. Then

$$
\phi \circ \gamma=\gamma \circ \varphi
$$

Therefore

$$
\mu \gamma(z)+c=\gamma(\varphi(z))
$$

for $z \in \mathbb{D}$. Now it follows that

$$
\mu \gamma^{\prime}(z)=\gamma^{\prime}(\varphi(z)) \cdot \varphi^{\prime}(z) .
$$


Since $\phi$ is an automorphism of $\Omega, \varphi$ is an automorphism of $\mathbb{D}$. Let $\beta=\varphi^{-1}(0)$. Then $\varphi(z)=\kappa(z-\beta) /(1-\bar{\beta} z)$ for some $|\kappa|=1$. Hence

$$
\frac{\gamma^{\prime}(z)}{\gamma^{\prime}(\varphi(z))}=\bar{\mu} \kappa \frac{1-|\beta|^{2}}{(1-\bar{\beta} z)^{2}}
$$

If $\psi(z)=\left(\frac{\gamma^{\prime}(z)}{\gamma^{\prime}(\varphi(z))}\right)^{1 / 2}$, then $\psi=\lambda \frac{K_{\beta}}{\left\|K_{\beta}\right\|}$, where $|\lambda|=1$ and $K_{\beta}(z)=1 /(1-\bar{\beta} z)$ is the reproducing kernel for $H^{2}$; i.e. if $f \in H^{2}$, then $\left\langle f, K_{\beta}\right\rangle=f(\beta)$. Thus $C_{\psi, \varphi}$ is a unitary operator on $H^{2}$ [1, Theorem 6], and by Lemma 2.2, $C_{\phi}$ is unitary on $H^{2}(\Omega)$.

Now suppose that $C_{\phi}$ is unitary on $H^{2}(\Omega)$. Then $C_{\psi, \varphi}$ is unitary on $H^{2}$, where

$$
\psi(z)=\left(\frac{\gamma^{\prime}(z)}{\gamma^{\prime}(\varphi(z))}\right)^{1 / 2}
$$

and $\varphi=\gamma^{-1} \circ \phi \circ \gamma$. If $C_{\phi}$ is invertible, then $\phi$ is an automorphism of $\Omega$; hence $\varphi$ is an automorphism of $\mathbb{D}$. If $\beta=\varphi^{-1}(0)$, then $\varphi(z)=\zeta(z-\beta) /(1-\bar{\beta} z)$ for some unimodular $\zeta$. Since $C_{\psi, \varphi}$ is unitary, $\psi=\lambda K_{\beta} /\left\|K_{\beta}\right\|$ for some unimodular $\lambda$ [1, Theorem 6]. Therefore

$$
\frac{\gamma^{\prime}(z)}{\gamma^{\prime}(\varphi(z))}=\lambda^{2} \frac{1-|\beta|^{2}}{(1-\bar{\beta} z)^{2}} .
$$

But $\bar{\zeta} \varphi^{\prime}(z)=\left(1-|\beta|^{2}\right) /(1-\bar{\beta} z)^{2}$, and thus

$$
\gamma^{\prime}(z)=\lambda^{2} \bar{\zeta} \gamma^{\prime}(\varphi(z)) \varphi^{\prime}(z)
$$

Therefore $\gamma^{\prime}(z)=\lambda^{2} \bar{\zeta}(\gamma(\varphi(z)))^{\prime}$, and by integrating both sides

$$
\gamma(z)=\lambda^{2} \bar{\zeta} \gamma(\varphi(z))+k
$$

Since $\gamma \circ \varphi=\phi \circ \gamma$, we have

$$
\gamma(z)=\lambda^{2} \bar{\zeta} \phi(\gamma(z))+k .
$$

The desired result follows by letting $w=\gamma(z)$.

\section{Finite ORDER}

Lemma 4.1. Suppose that $\mathcal{U}_{\Omega}$ is a finite group. Further, suppose that $\phi(w)=$ $\mu w+c$ and $\psi(w)=\mu w+d$. If $\phi, \psi \in \mathcal{U} \mathcal{A}_{\Omega}$, then $c=d$.

Proof. It is readily seen that

$$
\phi^{-1}(w)=\mu^{-1} w-c \mu^{-1} .
$$

If $\varphi=\psi \circ \phi^{-1}$, it follows that $\varphi \in \mathcal{U} \mathcal{A}_{\Omega}$ since $C_{\phi^{-1}} \circ C_{\psi} \in \mathcal{U}_{\Omega}$. Now

$$
\varphi(w)=\mu\left(\mu^{-1} w-c \mu^{-1}\right)+d=w-c+d .
$$

It is easy to see that $\varphi_{m}(w)=w+m(d-c)$ for any positive integer $m$. If $d-c \neq 0$, then $\left\{\varphi_{m}: m=1,2, \cdots\right\}$ is infinite and thus $G=\left\{C_{\varphi_{m}}: m=1,2, \cdots\right\}$ is also infinite. But $G \subseteq \mathcal{U}_{\Omega}$, and hence $d-c=0$.

Suppose that $n>1$ is an integer. The domain $\Omega$ is said to have $n$-fold symmetry about the point $p$ if $e^{2 \pi i / n}(\Omega-p)=\Omega-p$.

Theorem 4.2. If $\mathcal{U}_{\Omega}$ has finite order $n>1$, then $\Omega$ has $n$-fold symmetry and $\mathcal{U}_{\Omega}$ is isomorphic to $\mathbb{Z}_{n}$. 
Before giving the proof, we note that $n$-fold symmetry of $\Omega$ does not imply that $\mathcal{U}_{\Omega}$ has finite order. A simple example is when $\Omega$ is the unit disc $\mathbb{D}$, which has $n$-fold symmetry for every integer $n$. As previously noted, $\mathcal{U}_{\mathbb{D}} \cong(\mathbb{T}, \cdot)$. Another example is when $\Omega$ is a strip which has 2 -fold symmetry, but no higher symmetry. See Proposition 5.8 below.

Proof. If $C_{\phi} \in \mathcal{U}_{\Omega}$, then it follows from Theorem 3.2 that $\phi(w)=\mu w+c,|\mu|=1$. Since the order of $\mathcal{U}_{\Omega}$ is $n, C_{\phi}^{n}$ is the identity. But $C_{\phi}^{n}=C_{\phi_{n}}$ and $\phi_{n}(w)=$ $\mu^{n} w+\left(1+\mu+\cdots+\mu^{n-1}\right) c$. Thus $\mu^{n} w+\left(1+\mu+\cdots+\mu^{n-1}\right) c=w$, which gives $\mu^{n}=1$. Since $\phi$ is an automorphism, it follows that $\Omega$ has $n$-fold symmetry about $c /(1-\mu)$, the fixed point of $\phi$.

Let $G_{n}$ denote the group $\left\{e^{2 \pi i k / n}: k=0,1, \cdots, n-1\right\}$ under usual complex number multiplication. Define $\Pi: \mathcal{U}_{\Omega} \rightarrow G_{n}$ by

$$
\Pi\left(C_{\phi}\right)=\phi^{\prime}(0) \text {. }
$$

A straightforward computation shows that

$$
\Pi\left(C_{\phi_{1}} \circ C_{\phi_{2}}\right)=\mu_{1} \mu_{2}=\Pi\left(C_{\phi_{1}}\right) \Pi\left(C_{\phi_{2}}\right) .
$$

Thus $\Pi$ is a group homomorphism. From Lemma 4.1 it follows that $\Pi$ is one-to-one. Since $\mathcal{U}_{\Omega}$ and $G_{n}$ contain the same number of elements, $\Pi$ is onto as well. Hence $\Pi$ is an isomorphism. Clearly $G_{n}$ is isomorphic to $\mathbb{Z}_{n}$, and the result follows.

Example 4.3. Let $n>1$ be an integer. Let $\Omega$ be the interior of the regular $n$-sided polygon whose vertices are $1, e^{2 \pi i / n}, e^{2 \pi i 2 / n}, \cdots e^{2 \pi i(n-1) / n}$. Then $\mathcal{U}_{\Omega}$ is isomorphic to $\mathbb{Z}_{n}$.

Proof. It is easy to see that $f(w)=e^{2 \pi i \theta} w+c$ is an automorphism of $\Omega$ if and only if $\theta=m / n, m \in \mathbb{Z}$ and $c=0$. The desired result now follows from Theorem 3.2.

The following example gives a domain $\Omega$ with $\mathcal{U}_{\Omega}$ the trivial group, showing that order one is possible.

Example 4.4. Let $\Omega=\{x+i y, x>0, y>0\}$. Then $\mathcal{U}_{\Omega}$ is the trivial group.

It is easily seen that $f(w)=e^{2 \pi i \theta} w+c$ is an automorphism of $\Omega$ if and only if $f(w)=w$. Thus from Theorem 3.2 it follows that $\mathcal{U}_{\Omega}$ is the trivial group.

\section{INFINITE ORDER}

In this section we work under the assumption that $\mathcal{U}_{\Omega}$ is of infinite order.

Lemma 5.1. If $f(w)=w+\alpha$ and $g(w)=w+\beta$ are distinct automorphisms of $\Omega$, then the line through $\alpha$ and $\beta$ contains the origin.

Proof. Assume, contrary to the statement, that $\alpha$ and $\beta$ do not lie on the same line through the origin. Note that this implies $\alpha \beta \neq 0$. Next, observe that $f$ and $g$ are automorphisms of $\Omega$ if and only if $\widehat{f}(z)=z+1$ and $\widehat{g}(z)=z+\beta / \alpha$ are automorphisms of $\alpha^{-1} \Omega$. Thus we may assume that $\alpha=1$ and $\beta \notin \mathbb{R}$. We further assume that $\operatorname{Im} \beta>0$; if $\operatorname{Im} \beta<0$ the proof will be similar, except that the orientation of the curve $\Gamma_{N}$ below will be reversed.

Let $w_{0} \in \Omega$, so $f\left(w_{0}\right)=w_{0}+1 \in \Omega$ and $g\left(w_{0}\right)=w_{0}+\beta \in \Omega$ as well. Since $\Omega$ is path connected, there are smooth curves $\gamma$ from $w_{0}$ to $w_{0}+1$ and $\eta$ from $w_{0}$ to $w_{0}+\beta$ that lie in $\Omega$. Now fix

$$
R>\max \{\operatorname{diam}(\gamma), \operatorname{diam}(\eta)\},
$$


and choose an integer $N$ large enough so that the disc $B(0,2 R)$ with center 0 and radius $2 R$ is contained in the interior of the parallelogram $P_{N}$ with vertices $w_{0} \pm N \pm N \beta$.

Next recall from the end of $\S 2$ the definition of the iterates $\left\{f_{n}\right\}_{n \in \mathbb{Z}}$ and $\left\{g_{n}\right\}_{n \in \mathbb{Z}}$, and set

$$
\sigma=\sum_{n=-N}^{N-1} f_{n}(\gamma) \text { and } \lambda=\sum_{n=-N}^{N-1} g_{n}(\eta)
$$

Then the curve

$$
\Gamma_{N}=g_{-N}(\sigma)+f_{N}(\lambda)-g_{N}(\sigma)-f_{-N}(\lambda)=\sum_{j=1}^{4} \Gamma_{N j}
$$

is a closed curve in $\Omega$ that passes through the vertices of $P_{N}$.

Now let $\zeta \in B(0, R)$. Note that since $B(0,2 R)$ is in the interior of $P_{N}$, it follows from (5.1) that each $\Gamma_{N j}$ is contained in the complement of a half-plane that contains $B(0, R)$. Thus the increment of the argument of $z-\zeta$ over $\Gamma_{N j}$ satisfies

$$
0<\Delta \arg \left(z-\zeta, \Gamma_{N j}\right)<\pi .
$$

Therefore $0<\Delta \arg \left(z-\zeta, \Gamma_{N}\right)<4 \pi$, and hence $\Delta \arg \left(z-\zeta, \Gamma_{N}\right)=2 \pi$ since $\Gamma_{N}$ is a closed curve. Thus the winding number of $\Gamma_{N}$ about each point $\zeta \in B(0, R)$ is 1 , and it follows that $B(0, R) \subset \Omega$. Since the only assumption on $R$ was (5.1), this contradicts the standing assumption that $\Omega \neq \mathbb{C}$ and completes the proof.

Lemma 5.2. Suppose that $f(w)=\mu w+c$ and $g(w)=\mu w+d$ are both automorphisms of $\Omega$, where $\mu$ is unimodular. If $c \neq d$, then $\mu$ is 1 or -1 .

Proof. Let $w \in \Omega$. Then

$$
h(w)=f \circ g^{-1}(w)=w+c-d .
$$

Let

$$
p(w)=f \circ h \circ f^{-1}(w)=w+\mu(c-d) .
$$

Then $p$ and $h$ are automorphisms of $\Omega$. By Lemma 5.1$] c-d$ and $\mu(c-d)$ lie on the same line through the origin. Hence $\mu^{2}=1$.

We saw in Theorem 3.2 that $f \in \mathcal{U} \mathcal{A}_{\Omega}$ has the form $f(w)=\mu w+c$, and so extends to be an entire function. We will always consider $f$ extended in this way, so that even if $0 \notin \Omega$ we can write $f(0)=c$ and $f^{\prime}(0)=\mu$. This convention is used in the next definition and subsequently.

Definition 5.3. Let

$$
R_{\Omega}=\left\{f^{\prime}(0): f \in \mathcal{U} \mathcal{A}_{\Omega}\right\}
$$

Also, $R_{\Omega}=\{\mu: f(w)=\mu w+c, f$ is an automorphism of $\Omega,|\mu|=1\}$. Since the identity map is always in $\mathcal{U} \mathcal{A}_{\Omega}$ it is clear that $1 \in R_{\Omega}$. If $\lambda, \mu \in R_{\Omega}$, then there are $f, g \in \mathcal{U} \mathcal{A}_{\Omega}$ so that $f(w)=\lambda w+c$ and $g(w)=\mu w+d$. Since both $f \circ g, f^{-1} \in \mathcal{U} \mathcal{A}_{\Omega}$, it follows that $\lambda \mu \in R_{\Omega}$ and $\lambda^{-1} \in R_{\Omega}$. Hence $R_{\Omega}$ is a subgroup of $(\mathbb{T}, \cdot)$.

The set $R_{\Omega}$ can be infinite or finite. We leave the proof of the next lemma as an easy exercise.

Lemma 5.4. If $R_{\Omega}$ is infinite, then it is dense in the unit circle. 
Lemma 5.5. Suppose that $\mathcal{U}_{\Omega}$ is infinite. Then

(1) The following are equivalent:

(a) $R_{\Omega}$ is infinite.

(b) $\Omega$ is a disc.

(c) $R_{\Omega}=\mathbb{T}$.

(2) If $\Omega$ is not a disc, then $R_{\Omega} \subseteq\{1,-1\}$.

Proof. (a) $\Longrightarrow$ (b): Suppose that $R_{\Omega}$ is infinite. Choose $\mu_{1}, \mu_{2} \in R_{\Omega}$ such that $\left(\mu_{1} \mu_{2}\right)^{2} \neq 1$. Then there are $f, g \in \mathcal{U} \mathcal{A}_{\Omega}$ so that $f(w)=\mu_{1} w+c_{1}$ and $g(w)=$ $\mu_{2} w+c_{2}$. Now

$$
f \circ g(w)=\mu_{1} \mu_{2} w+\left(\mu_{1} c_{2}+c_{1}\right) \text { and } g \circ f(w)=\mu_{2} \mu_{1} w+\left(\mu_{2} c_{1}+c_{2}\right) .
$$

Since $f \circ g, g \circ f \in \mathcal{U} \mathcal{A}_{\Omega}$ and $\mu_{1} \mu_{2} \neq \pm 1$, it follows from Lemma 5.2 that $\mu_{1} c_{2}+c_{1}=$ $\mu_{2} c_{1}+c_{2}$. Thus

$$
\left(1-\mu_{2}\right) c_{1}=\left(1-\mu_{1}\right) c_{2}
$$

Since $R_{\Omega}$ is dense in $\mathbb{T}$ we can choose a dense sequence $\left\{\mu_{n}\right\}_{n=1}^{\infty}$ from $R_{\Omega}$ such that $\left(\mu_{i}\right)^{2} \neq 1$ and $\left(\mu_{i} \mu_{i+1}\right)^{2} \neq 1$. For each $\mu_{i}$ there is an automorphism $f(w)=$ $\mu_{i} w+c_{i}$. Since $\left(\mu_{i} \mu_{i+1}\right)^{2} \neq 1$, it follows from (5.3) that $c_{i} /\left(1-\mu_{i}\right)=c_{1} /\left(1-\mu_{1}\right)$ for $i=2,3, \cdots$. Let $p=c_{1} /\left(1-\mu_{1}\right)$ and

$$
E=\Omega-p .
$$

We first show that $\mu_{j} E \subseteq E$. If $v \in E$, then $v=w-p$ for some $w \in \Omega$. Now

$$
\mu_{j} v+p=\mu_{j} w-\frac{\mu_{j} c_{j}}{1-\mu_{j}}+\frac{c_{j}}{1-\mu_{j}}=\mu_{j} w+c_{j} .
$$

Since $\mu_{j} v \in E$ if and only if $\mu_{j} v+p \in \Omega$ it is readily seen that $\mu_{j} v \in E$.

Let $z \in \mathbb{C} \backslash E$. Since $z+p \in \mathbb{C} \backslash \Omega$ and $h(w)=\mu_{j} w+c_{j}$ is an automorphism of $\Omega$ that extends to $\mathbb{C}$, it follows that $\mu_{j}(z+p)+c_{j} \in \mathbb{C} \backslash \Omega$. Since $\mu_{j} z=\mu_{j}(z+p)+c_{j}-p$ it follows that $\mu_{j} z \in \mathbb{C} \backslash E$. Thus

$$
\left\{\mu_{j} z \mid j=1,2, \cdots\right\} \subseteq \mathbb{C} \backslash E .
$$

Note that $\mathbb{C} \backslash E$ is closed. The closure of $\left\{\mu_{j} z \mid j=1,2, \cdots\right\}$ is a circle centered at 0 which is contained in $\mathbb{C} \backslash E$. Also, $E$ is open and connected; hence $E$ is a disc centered at the origin. Thus $\Omega$ is a disc centered at $p$.

(b) $\Longrightarrow(\mathrm{c})$ : If $\Omega$ is a disc with center $p$, then $w \rightarrow e^{i \theta}(w-p)+p$ is an automorphism of $\Omega$ for all real $\theta$. Thus $R_{\Omega}=\mathbb{T}$.

The proof of $(\mathrm{c}) \Longrightarrow(\mathrm{a})$ is trivial.

Proof of (2): Since $\Omega$ is not a disc, from part (1) it follows that $R_{\Omega}$ is finite. If $|\lambda|=1, \lambda^{2} \neq 1$, then from Lemma 5.2 there is at most one $c^{\prime}$ such that $k(w)=$ $\lambda w+c^{\prime}$ is an automorphism of $\Omega$. But $\mathcal{U}_{\Omega}$ is infinite; thus there are infinitely many automorphisms of the form $f(w)=w+d$ or $g(w)=-w+k$. Let $\mu \in R_{\Omega}$. Then there is $h(w)=\mu w+c$ so that $h \in \mathcal{U} \mathcal{A}_{\Omega}$. Since

$$
h \circ f(w)=\mu w+(\mu d+c) \text { and } h \circ g(w)=-\mu w+(\mu k+c)
$$

it follows from Lemma 5.2 that $\mu^{2}=1$.

Part (1) of the previous lemma gives a complete description of $\Omega$ when $R_{\Omega}$ is infinite. Next we turn to the case that $R_{\Omega}$ is finite, so $R_{\Omega} \subseteq\{1,-1\}$ by Lemma 5.5. 
From Lemma 5.1 there is a unimodular constant $\lambda$ such that if $f(w)=w+c$ is in $\mathcal{U} \mathcal{A}_{\Omega}$, then $\bar{\lambda} c$ is real. Define

$$
S_{\Omega}=\left\{t \in \mathbb{R}: f_{t}(w)=w+t \lambda \text { is in } \mathcal{U} \mathcal{A}_{\Omega}\right\} .
$$

Since $f_{s} \circ f_{t}=f_{s+t},\left(S_{\Omega},+\right)$ is a subgroup of $(\mathbb{R},+)$.

Since we have assumed in this section that $\mathcal{U}_{\Omega}$ is infinite, there are infinitely many automorphisms of the form $f(w)=w+c$ or $g(w)=-w+d$. If there are infinitely many of the former, then it is clear that there exists $t \in S_{\Omega}$ with $t>0$. If there are infinitely many of the latter, take $g$ and $h$ to be two such automorphisms. Then

where $d_{1} \neq d_{2}$. But

$$
g(w)=-w+d_{1} \text { and } h(w)=-w+d_{2}
$$

$$
g \circ h(w)=w+\left(d_{1}-d_{2}\right)
$$

and again the set of $t>0$ with $t \in S_{\Omega}$ is nonempty. Let

$$
c_{\Omega}=\inf \left\{t>0: t \in S_{\Omega}\right\} \text {. }
$$

Lemma 5.6. Suppose that $R_{\Omega} \subseteq\{1,-1\}$.

(1) Suppose that $c_{\Omega}=0$. Then $\Omega$ is a half-plane or a strip and $S_{\Omega}=\mathbb{R}$. Moreover, $-1 \in R_{\Omega}$ if and only if $\Omega$ is a strip.

(2) $c_{\Omega}>0$ if and only if $S_{\Omega}=\left\{n \cdot c_{\Omega}: n \in \mathbb{Z}\right\}$.

Proof. Suppose that $c_{\Omega}=0$ and let $\lambda$ be as in the definition of $S_{\Omega}$. Let $l_{\lambda}$ be the line $\{\lambda t: t \in \mathbb{R}\}$. If $w \in \Omega$, then there is a $\delta>0$ such that $\Omega$ contains the line segment

$$
L_{w, \delta}=\{w+\lambda t:-\delta<t<\delta\} .
$$

Since $c_{\Omega}=0$, there is $t_{w} \in \mathbb{R}$ such that $0<\left|t_{w}\right|<\delta$ and $\phi(w)=w+\lambda t_{w}$ is an automorphism of $\Omega$. If $n \in \mathbb{Z}$, then $\phi_{n} \in \mathcal{U} \mathcal{A}_{\Omega}$; hence

$$
\bigcup_{n \in \mathbb{Z}} \phi_{n}\left(L_{w, \delta}\right) \subseteq \Omega
$$

But $\phi_{n}\left(L_{w, \delta}\right)=L_{w, \delta}+n \lambda t_{w}$. Thus $L_{w}=\bigcup_{n \in \mathbb{Z}} \phi_{n}\left(L_{w, \delta}\right)$ is a line that goes through $w$ and is parallel to $l_{\lambda}$.

Since $w$ is an arbitrary point in $\Omega$, it follows that $\bigcup_{w \in \Omega} L_{w} \subseteq \Omega$. Clearly $\Omega \subseteq$ $\bigcup_{w \in \Omega} L_{w} ;$ thus

$$
\Omega=\bigcup_{w \in \Omega} L_{w} .
$$

Since $\Omega \neq \mathbb{C}$ and is connected, it must be a strip or a half-plane. Thus $S_{\Omega}=\mathbb{R}$. Clearly $-1 \in R_{\Omega}$ implies that $\Omega$ is not a half-plane, and so must be a strip. Finally, the 2 -fold symmetry of a strip implies that $-1 \in R_{\Omega}$ if $\Omega$ is a strip.

Proof of (2): First suppose that $c_{\Omega}>0$. Since $\left(S_{\Omega},+\right)$ is a subgroup of $(\mathbb{R},+)$, if $c_{\Omega}>0$, then $S_{\Omega}$ has no point of accumulation and hence $c_{\Omega} \in S_{\Omega}$. It follows that $S_{\Omega}=\left\{n \cdot c_{\Omega}: n \in \mathbb{Z}\right\}$. Sufficiency easily follows since $S_{\Omega}$ is a nontrivial group.

If $\Omega$ is the upper half-plane, then $R_{\Omega}=\{1\}$. Next we will look at such regions.

Proposition 5.7. Suppose that $R_{\Omega}=\{1\}$. Then

(1) The following are equivalent:

(a) $c_{\Omega}=0$.

(b) $\Omega$ is a half-plane.

(c) $\mathcal{U}_{\Omega}$ is isomorphic to $(\mathbb{R},+)$.

(2) If $c_{\Omega}>0$, then $\mathcal{U}_{\Omega}$ is isomorphic to $(\mathbb{Z},+)$. 
Proof. (a) $\Longrightarrow$ (b) : It follows from (1) of Lemma 5.6 that $\Omega$ is a half-plane.

(b) $\Longrightarrow(\mathrm{c})$ : Since $\Omega$ is a half-plane there is a $\lambda \in \mathbb{C}$ so that the boundary of $\Omega$ is parallel to the line $l_{\lambda}=\{\lambda t: t \in \mathbb{R}\}$. If $t \in \mathbb{R}$, and $\phi(w)=w+t \lambda$, then $\phi \in \mathcal{U} \mathcal{A}_{\Omega}$. Define $\Pi: \mathcal{U}_{\Omega} \rightarrow(\mathbb{R},+)$ by

$$
\Pi\left(C_{\phi}\right)=\lambda^{-1} \phi(0) .
$$

It is readily seen that $\Pi$ is a bijection. If $f(w)=w+\lambda t_{1}, g(w)=w+\lambda t_{2}$, then $g \circ f(w)=w+\lambda\left(t_{1}+t_{2}\right)$. Since $C_{f} \circ C_{g}=C_{g \circ f}$ we get

$$
\Pi\left(C_{f} \circ C_{g}\right)=t_{1}+t_{2}=\Pi\left(C_{f}\right)+\Pi\left(C_{g}\right) .
$$

Thus $\mathcal{U}_{\Omega}$ is isomorphic to $(\mathbb{R},+)$.

$(\mathrm{c}) \Longrightarrow(\mathrm{a})$ : Suppose that $\mathcal{U}_{\Omega} \cong(\mathbb{R},+)$. Clearly $c_{\Omega} \geq 0$, and if $c_{\Omega}>0$, then part (2) yields that $\mathcal{U}_{\Omega} \cong(\mathbb{Z},+)$. Thus $c_{\Omega}=0$.

Proof of (2): This is clear from (2) of Lemma 5.6.

If $\Omega=\{z:-1<\operatorname{Im}(z)<1\}$, then $R_{\Omega}=\{1,-1\}$. Next we look at such regions.

Proposition 5.8. Suppose that $R_{\Omega}=\{1,-1\}$. Then

(1) The following are equivalent:

(a) $c_{\Omega}=0$.

(b) $\Omega$ is a strip.

(c) $\mathcal{U}_{\Omega}$ is isomorphic to $\{1,-1\} \rtimes \mathbb{R}$.

(2) If $c_{\Omega}>0$, then $\mathcal{U}_{\Omega}$ is isomorphic to $\{1,-1\} \rtimes \mathbb{Z}$.

Proof. (a) $\Longrightarrow(\mathrm{b})$ : The result follows from (1) of Lemma 5.6 .

(b) $\Longrightarrow(\mathrm{c})$ : Since $\Omega$ is a strip there is some $\lambda \in \mathbb{C}$ so that $\Omega$ is parallel to the line $l_{\lambda}=\{\lambda t: t \in \mathbb{R}\}$. Let $z_{0}$ be the point with minimum modulus such that

$$
-\Omega+z_{0}=\Omega \text {. }
$$

Therefore $s(w)=-w+z_{0} \in \mathcal{U} \mathcal{A}_{\Omega}$. For any $r(w)=-w+d \in \mathcal{U} \mathcal{A}_{\Omega}$ we have $r \circ s(w)=w+d-z_{0} \in \mathcal{U} \mathcal{A}_{\Omega}$; thus $d-z_{0}=\lambda t$ for some $t \in \mathbb{R}$. Hence if $f \in \mathcal{U} \mathcal{A}_{\Omega}$ and $f^{\prime}(0)=-1$, then $f(0)=z_{0}+\lambda t$. Thus, $C_{\phi} \in \mathcal{U}_{\Omega}$ if and only if $\phi(w)=w+\lambda t$ or $\phi(w)=-w+z_{0}+\lambda t, t \in \mathbb{R}$. Let $\Pi: \mathcal{U}_{\Omega} \rightarrow\{1,-1\} \rtimes \mathbb{R}$ be defined by

$$
\Pi\left(C_{\phi}\right)=\left(\phi^{\prime}(0), \lambda^{-1}\left(\phi(0)-\frac{z_{0}}{2}\left(1-\phi^{\prime}(0)\right)\right)\right) .
$$

It is easy to see that $\Pi$ is a bijection. If $\phi \in \mathcal{U} \mathcal{A}_{\Omega}$, then it can be written in the form $\phi(w)=\mu w+z_{0}(1-\mu) / 2+\lambda t$. Suppose that $h, s \in \mathcal{U} \mathcal{A}_{\Omega}$ with $h(w)=$ $\mu_{1} w+z_{0}\left(1-\mu_{1}\right) / 2+\lambda t_{1}$ and $s(w)=\mu_{2} w+z_{0}\left(1-\mu_{2}\right) / 2+\lambda t_{2}$. A straightforward computation yields that

$$
\Pi\left(C_{h} \circ C_{s}\right)=\left(\mu_{2} \mu_{1}, \mu_{2} t_{1}+t_{2}\right)=\Pi\left(C_{h}\right) * \Pi\left(C_{s}\right) .
$$

Thus, $\mathcal{U}_{\Omega} \cong\{1,-1\} \rtimes \mathbb{R}$.

(c) $\Longrightarrow$ (a) : Suppose that $\mathcal{U}_{\Omega} \cong\{1,-1\} \rtimes \mathbb{R}$. Clearly $c_{\Omega} \geq 0$, and if $c_{\Omega}>0$, then part (2) yields that $\mathcal{U}_{\Omega} \cong\{1,-1\} \rtimes \mathbb{Z}$. Thus $c_{\Omega}=0$.

Proof of (2): If $c_{\Omega}>0$, then from (2) of Lemma 5.6 we see that $S_{\Omega}=\left\{n \cdot c_{\Omega}\right.$ : $n \in \mathbb{Z}\}$. Recall that there is a unimodular $\lambda$ such that if $f \in \mathcal{U} \mathcal{A}_{\Omega}$ and $f^{\prime}(0)=1$, then $f(w)=w+\lambda t$ for some $t \in S_{\Omega}$. If $g(w)=-w+c$ and $h(w)=-w+d$ are automorphisms of $\Omega$, then $g \circ h(w)=w+c-d$ is also an automorphism of $\Omega$; hence $c-d=n \cdot c_{\Omega} \lambda$ for some $n \in \mathbb{Z}$. Thus, there is a $d_{0} \in \mathbb{C}$ such that if 
$h \in \mathcal{U} \mathcal{A}_{\Omega}$ and $h^{\prime}(0)=-1$, then $h(w)=-w+d_{0}+m \cdot c_{\Omega} \lambda$ for some $m \in \mathbb{Z}$. Let $\Pi: \mathcal{U}_{\Omega} \rightarrow\{1,-1\} \rtimes \mathbb{Z}$ be defined by

$$
\Pi\left(C_{\phi}\right)=\left(\phi^{\prime}(0),\left(c_{\Omega} \lambda\right)^{-1}\left(\phi(0)-\frac{d_{0}}{2}\left(1-\phi^{\prime}(0)\right)\right)\right) .
$$

Then it is easy to see that $\Pi$ is an isomorphism.

Proposition 5.9. The group $(\mathbb{T}, \cdot)$ is isomorphic to $\mathcal{U}_{\Omega}$ if and only if $\Omega$ is a disc.

Proof. First suppose that $\Omega$ is a disc centered at the origin. It is readily seen that $f \in \mathcal{U} \mathcal{A}_{\Omega}$ if and only if $f(w)=e^{2 \pi i \theta} w$ for some $\theta \in \mathbb{R}$ (Theorem 3.2). The map $\Pi: \mathcal{U}_{\Omega} \rightarrow(\mathbb{T}, \cdot)$ defined by

$$
\Pi\left(C_{\phi}\right)=\phi^{\prime}(0)
$$

shows that $\mathcal{U}_{\Omega}$ is isomorphic to $(\mathbb{T}, \cdot)$. If $\Omega$ is a disc centered at $p$, then the same proof works with the domain $\Omega-p$.

Next we prove the other direction. Suppose that $\mathcal{U}_{\Omega}$ is isomorphic to $(\mathbb{T}, \cdot)$. From

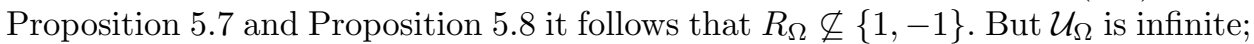
hence the desired result follows from Lemma 5.5

We summarize all the results in the theorem below.

Theorem 5.10. If $\mathcal{U}_{\Omega}$ is infinite, then it is isomorphic to one of the following groups:
(1) $(\mathbb{T}, \cdot)$
(2) $(\mathbb{R},+)$,
(3) $(\mathbb{Z},+)$
(4) $\{1,-1\} \rtimes \mathbb{R}$,
(5) $\{1,-1\} \rtimes \mathbb{Z}$.

Proof. If $R_{\Omega}$ is infinite, then from Lemma 5.5 and Proposition 5.9 it follows that $\mathcal{U}_{\Omega} \cong(\mathbb{T}, \cdot)$.

If $R_{\Omega}$ is not infinite, then from Lemma 5.5 it is contained in $\{1,-1\}$. Notice that 1 is always contained in $R_{\Omega}$. Thus $R_{\Omega}=\{1\}$ or $R_{\Omega}=\{1,-1\}$. If $R_{\Omega}=\{1\}$, then Proposition 5.7 yields that $\mathcal{U}_{\Omega}$ is isomorphic to either $(\mathbb{R},+)$ or $(\mathbb{Z},+)$. If $R_{\Omega}=\{1,-1\}$, then from Proposition 5.8 it follows that $\mathcal{U}_{\Omega} \cong\{1,-1\} \rtimes \mathbb{R}$ or $\mathcal{U}_{\Omega} \cong\{1,-1\} \rtimes \mathbb{Z}$.

In some of these cases we can give a precise description of $\Omega$.

Theorem 5.11. If $\mathcal{U}_{\Omega}$ is infinite, then the following are true.

(1) $\mathcal{U}_{\Omega} \cong(\mathbb{T}, \cdot)$ if and only if $\Omega$ is a disc.

(2) $\mathcal{U}_{\Omega} \cong(\mathbb{R},+)$ if and only if $\Omega$ is a half-plane.

(3) $\mathcal{U}_{\Omega} \cong\{1,-1\} \rtimes \mathbb{R}$ if and only if $\Omega$ is a strip.

Proof. Proposition 5.9 yields (1); (2) follows from Proposition 5.7 and Proposition 5.8 gives (3).

However, many different $\Omega$ can lead to $(\mathbb{Z},+)$ or $\{1,-1\} \rtimes \mathbb{Z}$. We close the paper with a few examples.

Example 5.12. Let

$$
\Omega=\{x+i y: y>\sin (x)\}
$$

Then $\mathcal{U}_{\Omega} \cong(\mathbb{Z},+)$. 
It is easy to see that $f \in \mathcal{U} \mathcal{A}_{\Omega}$ if and only if $f(w)=w+2 \pi n, n \in \mathbb{Z}$. Thus $R_{\Omega}=\{1\}$, and the result follows from Proposition 5.7 .

Example 5.13. Let

$$
\Omega=\{x+i y: 1+\sin (x)>y>\sin (x)\} .
$$

Then $\mathcal{U}_{\Omega} \cong\{1,-1\} \rtimes \mathbb{Z}$.

It is readily seen that $f \in \mathcal{U} \mathcal{A}_{\Omega}$ if and only if $f(w)=w+2 \pi n, n \in \mathbb{Z}$, or $f(w)=-w+i+(2 n) \pi, n \in \mathbb{Z}$. Hence $R_{\Omega}=\{1,-1\}$, and Proposition 5.8 yields the desired result.

Example 5.14. For $k \in \mathbb{Z}$ define $N_{k}^{+}=\{2 k+y i: 1 / 2 \leq y<1\}$ and $N_{k}^{-}=$ $\{(2 k+1)+y i:-1<y \leq-1 / 2\}$. Let

$$
\Omega=\{z:-1<\operatorname{Im}(z)<1\} \backslash \bigcup_{k \in \mathbb{Z}}\left(N_{k}^{+} \cup N_{k}^{-}\right) .
$$

Then $\mathcal{U}_{\Omega} \cong\{1,-1\} \rtimes \mathbb{Z}$.

Clearly $f \in \mathcal{U} \mathcal{A}_{\Omega}$ if and only if $f(w)=w+2 n$ or $f(w)=-w+1+2 n, n \in \mathbb{Z}$. Thus $R_{\Omega}=\{1,-1\}$, and the result follows from Proposition 5.8 .

\section{REFERENCES}

[1] Paul S. Bourdon and Sivaram K. Narayan, Normal weighted composition operators on the Hardy space $H^{2}(\mathbb{U})$, J. Math. Anal. Appl. 367 (2010), no. 1, 278-286, DOI 10.1016/j.jmaa.2010.01.006. MR2600397(2011a:47049)

[2] Carl C. Cowen and Barbara D. MacCluer, Composition operators on spaces of analytic functions, Studies in Advanced Mathematics, CRC Press, Boca Raton, FL, 1995. MR.1397026 (97i:47056)

[3] Peter L. Duren, Theory of $H^{p}$ spaces, Pure and Applied Mathematics, Vol. 38, Academic Press, New York, 1970. MR0268655 (42 \#3552)

[4] Gajath Gunatillake, Invertible weighted composition operators, J. Funct. Anal. 261 (2011), no. 3, 831-860, DOI 10.1016/j.jfa.2011.04.001. MR2799582(2012h:47055)

[5] Sam Elliott and Michael T. Jury, Composition operators on Hardy spaces of a half-plane, Bull. Lond. Math. Soc. 44 (2012), no. 3, 489-495, DOI 10.1112/blms/bdr110. MR2966995

[6] Valentin Matache, Composition operators on Hardy spaces of a half-plane, Proc. Amer. Math. Soc. 127 (1999), no. 5, 1483-1491, DOI 10.1090/S0002-9939-99-05060-1. MR.1625773 (99h:47038)

[7] Joel H. Shapiro and Wayne Smith, Hardy spaces that support no compact composition operators, J. Funct. Anal. 205 (2003), no. 1, 62-89, DOI 10.1016/S0022-1236(03)00215-5. MR:2020208 (2004h:30041)

[8] Wayne Smith and Alexander Volberg, A conformal mapping example (English, with English and French summaries), C. R. Math. Acad. Sci. Paris 349 (2011), no. 9-10, 511-514, DOI 10.1016/j.crma.2011.04.010. MR2802915(2012d:30014)

Department of Mathematics and Statistics, American University of Sharjah, UAE

E-mail address: gajathg@gmail.com

E-mail address: mgunatillake@aus.edu

Department of Mathematics, University of Hawail, Honolulu, Hawail 96822

E-mail address: jovovic@math.hawaii.edu

Department of Mathematics, University of Hawait, Honolulu, Hawail 96822

E-mail address: wayne@math.hawaii.edu 